\title{
Molecular length distribution and the formation of smectic phases
}

\author{
Nadia Kapernaum ${ }^{1}$, C. Scott Hartley ${ }^{2}$, Jeffrey C. Roberts ${ }^{2}$, \\ Robert P. Lemieux ${ }^{2}$ and Frank Giesselmann ${ }^{* 1}$
}

Open Access

\author{
Full Research Paper \\ Address: \\ ${ }^{1}$ Institut für Physikalische Chemie, Universität Stuttgart, D-70569 \\ Stuttgart, Germany and 2Department of Chemistry, Queen's \\ University, Kingston, Ontario, Canada \\ Email: \\ Frank Giesselmann* - f.giesselmann@ipc.uni-stuttgart.de \\ ${ }^{*}$ Corresponding author \\ Keywords: \\ bidispersity; liquid crystals; phase diagrams; smectic phases; \\ structure and dynamics
}

\author{
Beilstein Journal of Organic Chemistry 2009, 5, No. 65 \\ doi:10.3762/bjoc.5.65 \\ Received: 22 July 2009 \\ Accepted: 09 November 2009 \\ Published: 13 November 2009 \\ Guest Editor: S. Laschat \\ (c) 2009 Kapernaum et al; licensee Beilstein-Institut. \\ License and terms: see end of document.
}

\begin{abstract}
The phase diagrams of two mixtures of chemically similar smectogenic mesogens strongly differing in molecular length were investigated. In these mixtures the nematic phase present in the pure short mesogen disappeared rapidly on the addition of the longer mesogen, while the smectic state was preserved. In the smectic state the smectic A phase was the much more stable phase as the smectic $\mathrm{C}$ phase disappeared quite rapidly as well. In these compounds the loss of the smectic $\mathrm{C}$ phase is accompanied by a decrease in smectic translational order and very small tilt angles. This leads to a concentration induced smectic $\mathrm{C}$ to smectic $\mathrm{A}$ transition. Thus smectic A seems to be the most stable phase to accommodate mesogenic molecules of substantially different length. These surprising results are of general interest for the understanding of the structure and dynamics of smectic phases, as the structure of these bidisperse smectics is signified by extensive out-of-layer fluctuations.
\end{abstract}

\section{Introduction}

The classical (and highly successful) approach to systematically tailor liquid crystal materials for specific applications is the formulation of optimised mixtures consisting of several mesogenic compounds and non-mesogenic additives such as chiral dopants or UV-stabilizers. While the design of nematic mixtures is highly developed and widely applied, far less is known about the mixing of smectics and the particular effects thereof. In principle the mixing of different kind of mesogens can lead to a phase behaviour that differs completely from that of the pure compounds. This effect is even amplified in mixtures of mesogens with strongly differing molecular structure. In this paper we report a systematic study with mesogens strongly differing in molecular length.

We recently discovered that the electroclinic effect of a chiral smectic $\mathrm{A}^{*}\left(\mathrm{SmA}^{*}\right)$ material (consisting of a phenylpyrimidine host and $4 \mathrm{~mol} \%$ of a chiral atropisomeric dopant) was amplified by a factor of three after adding only $5 \%$ of another homo- 
logous phenylpyrimidine, the molecular length of which was about twice the length of the host molecule [1]. This remarkable electroclinic effect amplification stimulated a more general investigation of how the mixing of smectogenic homologues differing only in molecular lengths (and thus making the distribution of molecular length extremely bimodal) changes the structure and properties of the nematic and the SmA phases and the (possible) tilting transition to smectic $\mathrm{C}(\mathrm{SmC})$.

As a general first approximation calamitic mesogens are considered as rigid rods. This means they are treated as long and thin hard spherocylinders [2]. The justification for this rather crude approximation, which neglects the flexibility of the alkyl side chains, is the general observation that the thickness of e.g. a smectic A layer (as observed in X-ray diffraction) is only slightly smaller than the fully extended length of the constituting mesogenic molecules. In a naive model we now consider the liquid crystalline phase behaviour of mixtures of two types of these hard spherocylinders, which exhibit the same diameter but their lengths are differing by a factor of two. In this naive model, the nematic phase is expected - due to the absence of translational order - to be the ideal liquid crystalline (LC) phase for accommodating molecules of substantially different lengths. On the other hand the smectic phases - due to their layer structure - seem to be unsuited to accommodate molecules of different lengths. Comparing SmC and SmA, the SmC phase might be better since it allows different tilt angles to fit different molecular lengths into a smectic layer with fixed spacing (Figure 1).

The actual results received in this study are indeed completely reverse to the naive picture drawn above. The bimodal length distribution of smectogenic molecules favours the formation of non-tilted SmA phases at the expense of nematic or tilted SmC phases.

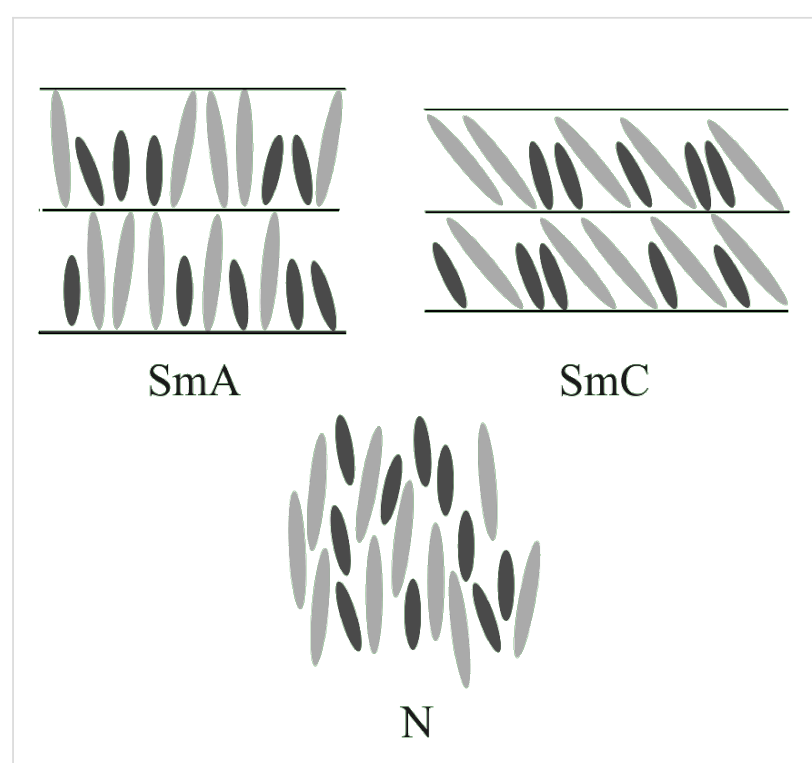

Figure 1: Structure of different liquid crystalline phases build with two kinds of hard spherocylinders with strongly differing lengths.

\section{Results and Discussion}

Scheme 1 and Figure 2 show the liquid crystalline materials we used in this experimental study. As the long component we chose the phenylpyrimidine PhP14 (2-[4-(tetradecyloxy)phenyl]-5-(tetradecyloxy)pyrimidine) [1], where the aromatic core is substituted symmetrically with two alkoxy chains each with 14 methylene units. It exhibits a molecular length of 45.5 $\AA$. For the short compound we used either the phenylpyrimidine 2PhP (2-[4-(butyloxy)phenyl]-5-(octyloxy)pyrimidine) [1] or the phenylpyridazine $6 \mathbf{P h P z}$ (6-[4-(butyloxy)phenyl]-3(octyloxy)pyridazine) $[3,4]$. Both were asymmetrically substituted with two alkoxy chains with four and eight methylene units, respectively. Their molecular length is $25.6 \AA$. This leads to a difference in lengths of a factor of the order of two.

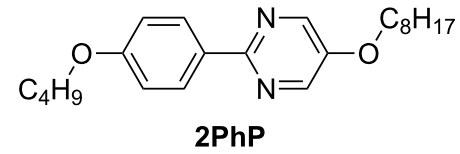

Cr 59.4 SmC 87.6 SmA 96.8 N 98.6 I

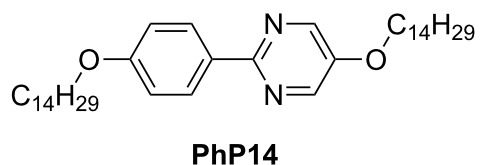

Cr 75.3 SmC 102.8 I

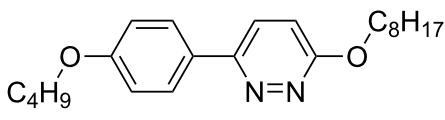

6PhPz

Cr 94.9 SmC 104.1 I

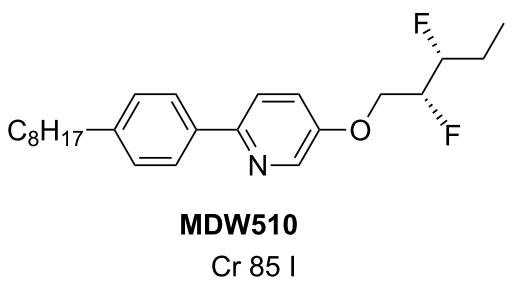



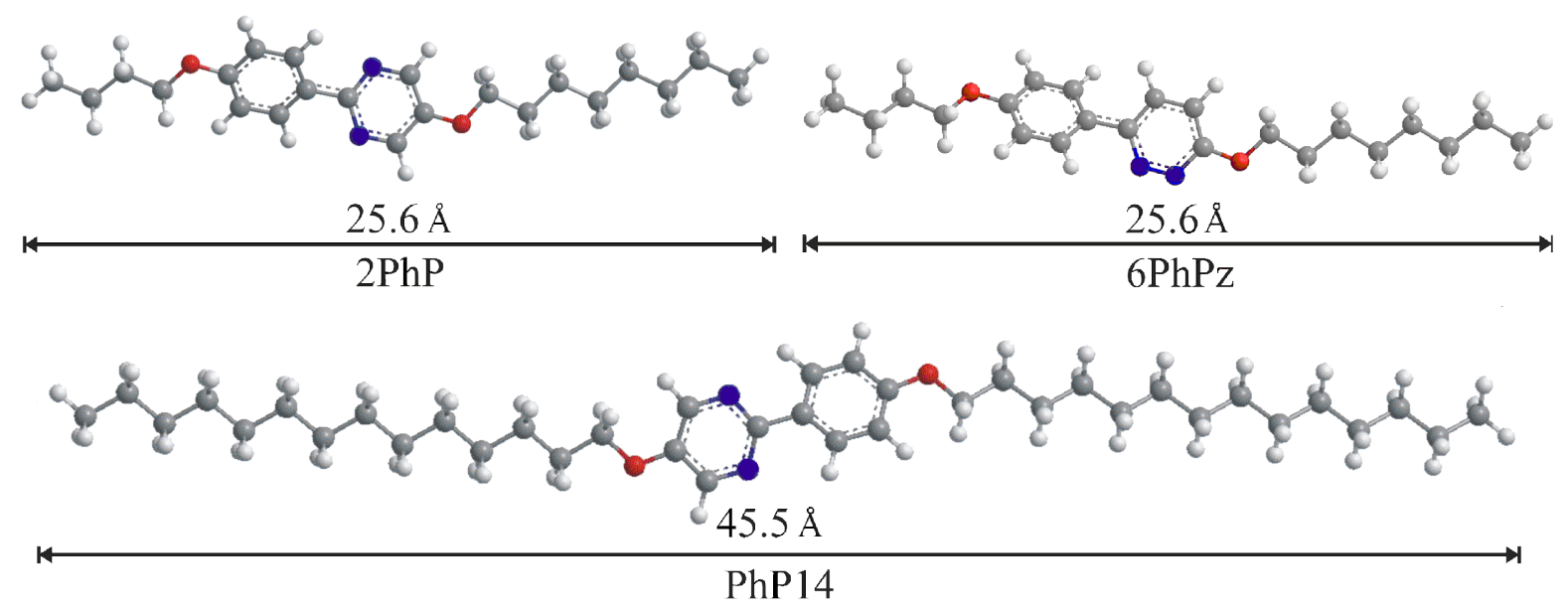

Figure 2: Molecular structure of the mesogens PhP14, 6PhPz and 2PhP differing in molecular lengths by a factor of approximately two. The molecular lengths were determined from the most extended conformers, after optimizing their energy using molecular modelling with MOPAC/AM1.

First we investigated the phase diagram of the system $\mathbf{2 P h P /}$ PhP14 which is shown in Figure 3. This phase diagram shows completely different behaviour from that expected from the naive model. In particular we observed no indication of destabilization of the smectic state. We could even observe a eutectic point at $x_{\mathrm{PhP} 14}=0.075$, where the temperature range of the smectic state is broadened. The nematic state on the other hand disappears rapidly with increasing mole fraction $x_{\mathrm{PhP} 14}$ and is already lost at a mole fraction $x_{\mathrm{PhP} 14}$ of 0.3 . In the smectic state SmA turns out to be the much more stable phase. $\mathrm{SmC}$ disappears quite rapidly and $\mathrm{SmA}$ is the dominating phase in the phase diagram even though $\mathrm{SmC}$ is the dominating $\mathrm{LC}$ phase of 2PhP and the only LC phase of PhP14. Taking into account that the average alkyl chain length increases with

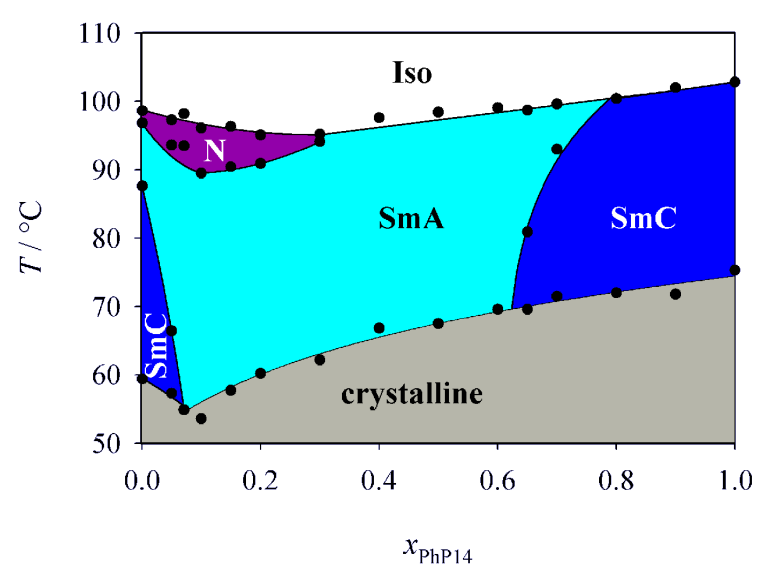

Figure 3: Phase diagram of the system 2PhP/PhP14. Over a broad temperature and concentration range only the SmA phase is stable. increasing $x_{\mathrm{PhP} 14}$, this behaviour is to some extent analogous to the well known mesomorphism in homologous series where nematic is the dominating mesophase for short-length homologues, SmA for medium-length homologues and $\mathrm{SmC}$ for longer homologues (see e.g. [1]).

For all mixtures small angle X-ray scattering (SAXS) measurements were performed. Figure 4 shows the layer spacing in the SmA phase at $T=T_{\mathrm{c}}$ in dependence on the mole fraction. A linear correlation between the layer spacing and the mole fraction is found. This linear dependence shows that the system 2PhP/PhP14 follows the Diele additivity rule [5]. It says that the layer spacing of a mixture can be calculated as: $d_{\mathrm{Mix}}=d_{\mathrm{A}}$ $x_{\mathrm{A}}+d_{\mathrm{B}} x_{\mathrm{B}}$, where $d_{\mathrm{Mix}}, d_{\mathrm{A}}$ and $d_{\mathrm{B}}$ denote the layer spacings of

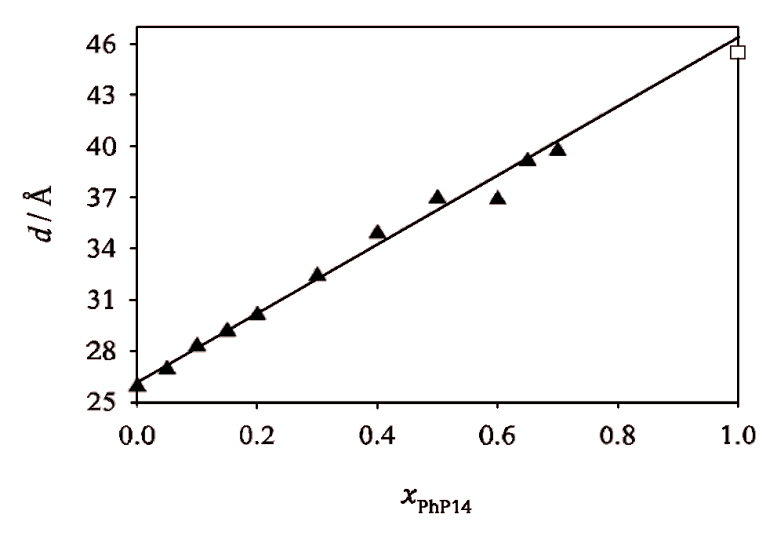

Figure 4: The layer spacing in the SmA phase at $T=T_{\mathrm{C}}$ vs mole fraction for the system 2PhP/PhP14 (filled triangles) and calculated value of the molecular length for pure PhP14 from molecular modelling (open square). 
the mixture, the pure compound $\mathrm{A}$ and the pure compound $\mathrm{B}$, respectively and $x_{\mathrm{A}}$ and $x_{\mathrm{B}}$ the mole fractions of compound $\mathrm{A}$ and $\mathrm{B}$, respectively. From this equation the layer spacing of a hypothetical SmA phase of pure PhP14 can be estimated. The extrapolated value of $46.4 \AA$ corresponds quite well to the value of $45.5 \AA$ we obtained for the extended length of the PhP14 molecule from molecular modelling studies (see Figure 2). For 2PhP the experimental $d$-value from SAXS $(25.9 \AA)$ also agrees very well with the extended length of the molecule $(25.6 \AA)$. This agreement between the experimental $d$-values and the extended molecular lengths for both pure compounds justifies to a certain extent the application of the spherocylinder model in these cases.

Figure 5 gives an overview of the layer spacings of pure $\mathbf{2 P h P}$ and of the mixtures with 5\%,65\% and 70\% PhP14 in 2PhP, respectively. The reduced layer spacing (calculated from the measured layer spacing divided by the layer spacing of the SmA phase) is plotted vs the temperature difference $T-T_{\mathrm{c}}$ relative to the phase transition temperature from $\mathrm{SmA}$ to $\mathrm{SmC}$. The pure compound 2PhP shows a 'common' behaviour of the layer spacing with significant layer shrinkage due to the molecular tilt in $\mathrm{SmC}$ of about $7 \%$ at $T-T_{\mathrm{c}}=20 \mathrm{~K}$. In the mixture with $5 \%$ PhP14 the layer shrinkage in the SmC phase is reduced to only $5 \%$. Very small layer shrinkage of only $1 \%$ was found for the mixture with $70 \%$ PhP14. And for the mixture with $65 \%$ PhP14 no layer shrinkage at all could be found, although by polarizing microscopy the broken fan-shaped texture of a SmC phase was clearly observed (see Figure 6).

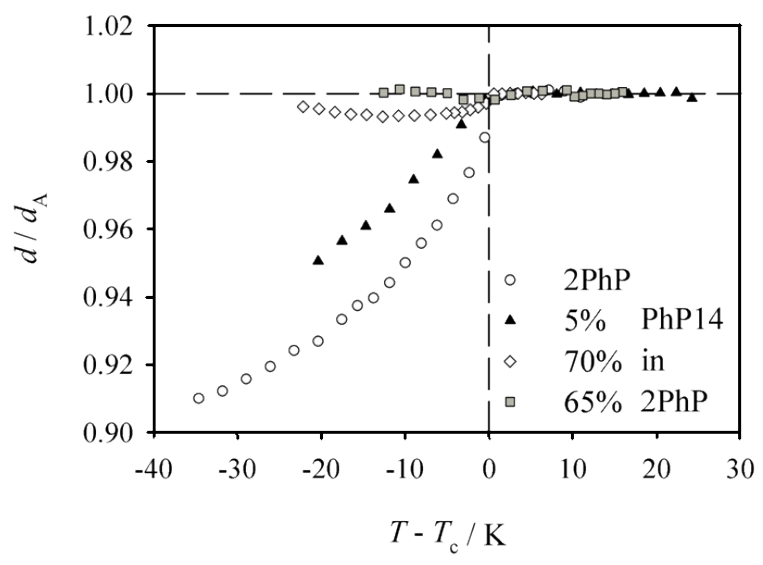

Figure 5: Reduced layer spacing for the mixtures which exhibit SmA and SmC phases in the system 2PhP/PhP14. Pure 2PhP shows quite normal layer shrinkage of $7 \%$ at $T-T_{\mathrm{C}}=-20 \mathrm{~K}$ (open circles). The addition of $5 \%$ of PhP14 reduces the layer shrinkage to only $5 \%$ at $T-T_{\mathrm{C}}=$ $-20 \mathrm{~K}$ (filled triangles). For the mixtures with excess of the long homologue $\mathrm{PhP} 14$ the layer shrinkage is dramatically reduced. The $70 \%$ mixture exhibits a layer shrinkage of only $1 \%$ at $T-T_{\mathrm{C}}=-20 \mathrm{~K}$ and for the mixture with $65 \%$ no layer shrinkage below the SmA to SmC phase transition could be observed at all.
To gain a deeper understanding of these smectic phases the optical tilt angle of the mixtures was measured in the corresponding ferroelectric $\mathrm{SmC}^{*}$ state [6] (see Figure 7) after addition of $4 \mathrm{~mol} \%$ of the chiral dopant MDW510 $((R, R)$-2-[4(octyloxy)phenyl]-5-(2,3-difluorohexyloxy)pyridine) [7,8]. The pure compound PhP14 exhibits the highest tilt angles, with a quite regular value of about $27^{\circ}$. The addition of more and more of the longer molecules reduces the SmC-tilt stepwise until only a SmA phase is left. This system thus shows a concentration induced $\mathrm{SmC}$ to SmA phase transition and therefore opens the possibility to design SmC phases with very small tilt angles [9]. These tilt angles are in good agreement with the tilt angles calculated from the X-ray layer shrinkage after: $\theta=\cos ^{-1}\left(d_{\mathrm{C}} /\right.$ $d_{\mathrm{A}}$ ). This correlation between the optical and X-ray tilt angles shows that, even if the layer shrinkage is very small, the mixtures do not necessarily exhibit the so-called 'de Vries-type' behaviour [10].

For a deeper insight into the quality of molecular ordering inside the smectic layer structure the translational order para-

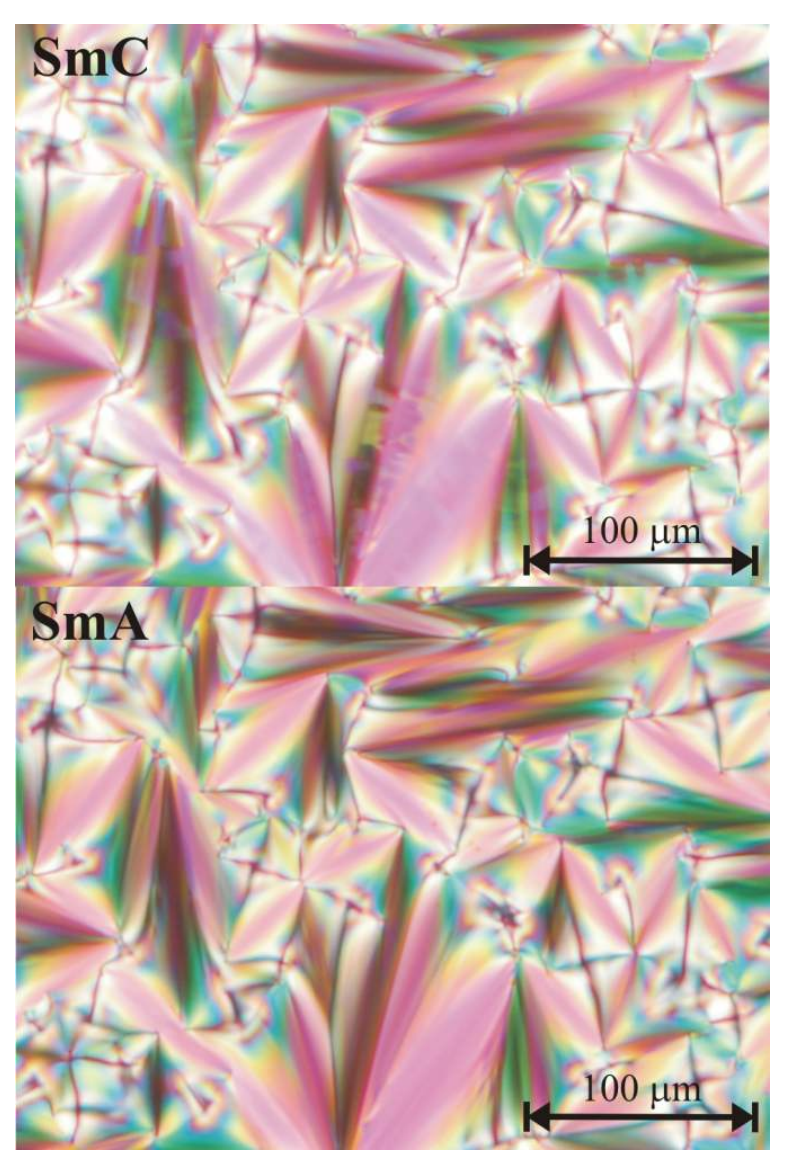

Figure 6: Textures of the mixture with 65\% PhP14 in 2PhP as observed in the polarizing microscope. The upper part shows the broken fan-shaped texture of the SmC phase at $T=75^{\circ} \mathrm{C}$. In the lower part the fan-shaped texture of SmA is observed at $T=86^{\circ} \mathrm{C}$. 


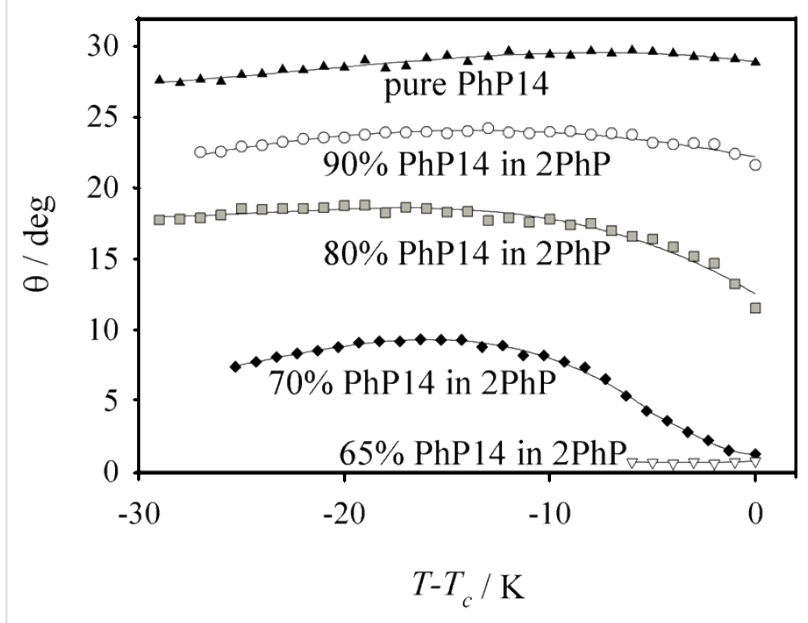

Figure 7: Tilt angle vs temperature difference to the phase transition temperature for pure PhP14 (filled triangles), 90\% (open circles), $80 \%$ (gray filled squares), $70 \%$ (filled diamonds) and $65 \%$ (open triangles) $\mathrm{PhP} 14$ in 2PhP. The tilt angle is reduced successively with increasing mole fraction of the short component $2 \mathrm{PhP}$. In this system a concentration-induced phase transition from SmC to SmA occurs.

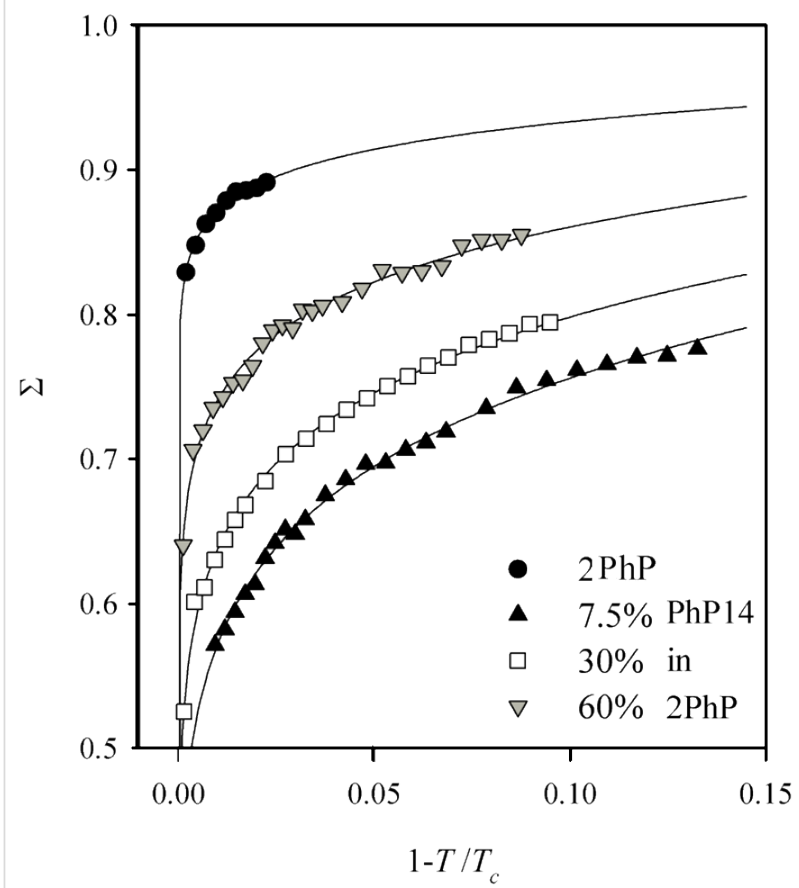

Figure 8: The translational order parameter $\Sigma$ in the SmA phase is plotted vs the reduced temperature $1-T / T_{\mathrm{C}}$ for the pure component 2PhP and the mixtures with $7.5 \%, 30 \%$ and $60 \%$ PhP14 in 2PhP, respectively. The pure $\mathbf{2} \mathbf{P h P}$ shows the highest order parameters of about 0.9 . The value of $\Sigma$ is reduced after the addition of $7.5 \%$ of PhP14 to about 0.7 and increases again slowly by the addition of more PhP14 for the mixture with $30 \%$ PhP14 until it reaches a value of 0.8 for the $60 \%$ mixture. meter $\Sigma[11]$ of the SmA phases was determined by a method previously described in [12]. The translational order parameter $\Sigma$ gives a measure for the quality of smectic layering. It is defined as the amplitude of the density wave arising from the 1D-periodic smectic layer structure.

Figure 8 shows the smectic order parameters $\Sigma$ of the SmA phase for the pure component $\mathbf{2} \mathbf{P h P}$ and for the mixtures with 7.5\%, 30\% and 60\% PhP14 in 2PhP, respectively. Pure 2PhP forms a SmA phase with a high degree of smectic order. It exhibits a smectic order parameter of about 0.9 . By adding a small amount of the other component the translational order is considerably reduced. For the mixture with $7.5 \%$ PhP14 (the eutectic mixture, see Figure 3) $\Sigma$ is in the range of about 0.7 and further increases on the addition of more of PhP14. It shows a value of about 0.75 for the $30 \%$ mixture and finally reaches a value of about 0.8 in the $60 \%$ mixture. The smallest value for $\Sigma$ was observed at that point in the phase diagram where the $\mathrm{SmC}$ phase is lost. And the re-entering of the SmC phase into the phase diagram is preceded by a recovery of the smectic order.

To check whether these findings can be generalized, we investigated another phase diagram of two strict $\mathrm{SmC}$ mesogens with a length ratio of 1:2. The mixing of two strict $\mathrm{SmC}$ mesogens should lead to a phase diagram where only SmC phases emerge.

Figure 9 shows the phase diagram of the system $6 \mathbf{P h P z} / \mathbf{P h P 1 4}$. This phase diagram is very similar to the one of the system 2PhP/PhP14. Although the two pure compounds show just $\mathrm{SmC}$ phases, the SmC phases disappear rapidly with increasing $x_{\mathrm{PhP} 14}$ and SmA replaces SmC. However, the stability of the smectic state is preserved again.

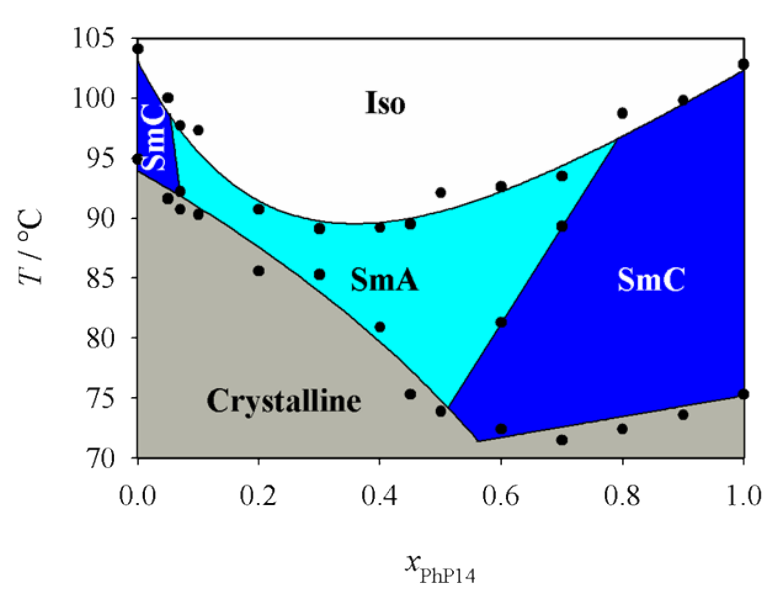

Figure 9: Phase diagram of the system 6PhPz/PhP14. Over a broad temperature region only the SmA phase is stable, although the two pure compounds exhibit only SmC phases. 
The reduced layer spacing of the mixtures with $60 \%$ and $70 \%$ PhP14 in 6PhPz can be seen in Figure 10. Both mixtures show small layer shrinkage of only $2 \%$ for the $70 \%$ mixture and $0.7 \%$ for the $60 \%$ mixture, respectively. This small layer shrinkage might also be related to small tilt angles in the corresponding SmC phases. The smectic order parameters $\Sigma$ of the mixtures could not be compared with those of the pure compounds, as the method described in [12] only applies to SmA phases whereas the pure components exhibit SmC phases only.

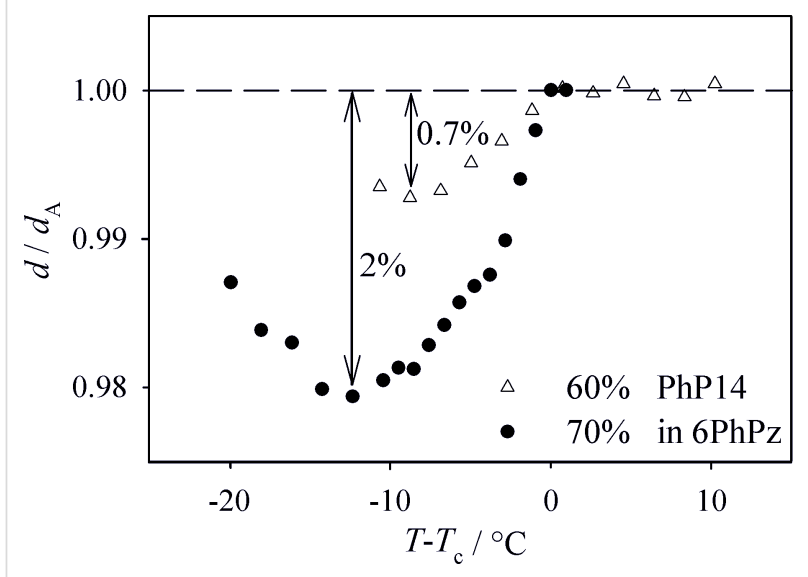

Figure 10: Reduced layer spacing vs temperature difference to the phase transition temperature for the mixtures with $60 \%$ and $70 \%$ PhP14 in 6PhPz. The layer shrinkage is only $2 \%$ for the $70 \%$ mixture and $0.7 \%$ for the $60 \%$ mixture, respectively.

\section{Conclusions}

Our investigations on two different phase diagrams of mesogenic molecules with chemically similar cores but with length ratios in the order of 2:1 led us to the following general results:

- When the short-length compound exhibits a nematic phase, the nematic phase disappears quickly with increasing mole fraction of the compound with greater molecular length.

- Surprisingly, the temperature range of the smectic states is preserved. It even becomes broader in some cases. Nevertheless the quality of smectic layering is lowered.

- We observed that over a broad temperature range the $\mathrm{SmC}$ phase is completely lost even though $\mathrm{SmC}$ is the dominating phase in the pure compounds. Instead of SmC, now the non-tilted SmA phase temperature range is broadened.

- In the regimes before $\mathrm{SmC}$ phases disappear, the mixtures show exceptionally small tilt angles (maximum tilt $<10^{\circ}$ over about $20 \mathrm{~K}$ ).
These results open pathways to a systematic design of interesting new low-tilt SmC materials.

To learn more about the ordering in smectic phases we compared our results with the work of Koden et al. [13]. They investigated several bidisperse mixtures of molecules with strongly differing molecular cores, but the same molecular length. In their mixtures they observed the same behaviour as in our mixtures. The nematic phases disappeared rapidly, as well as the smectic $\mathrm{C}$ phase, while the smectic A phase was the only stable phase over the whole phase diagram. They also found a dramatic decrease of the tilt angle in the remaining SmC phases, until the SmC phases disappeared at a concentration-induced phase transition to SmA.

These quite counterintuitive results are of general interest for the understanding of the structure and dynamics of smectic phases. Several theoretical approaches have been made to predict the behaviour of bidisperse mixtures [14-18]. They analyzed the stability of smectic A, nematic and isotropic phases by theoretical models of the Onsager-type in dependence on the composition of mesogens with different length and aspect ratios. They indeed predicted the occurrence of smectic phases in bidisperse mixtures for certain length and aspect ratios. In all these theoretical works however a stabilization of the nematic state at the expense of the smectic state was found. This is contradictory to the experimental findings, as our findings showed a stabilization of the smectic state while the nematic phase disappeared completely. The existing theories thus do not describe these results correctly. Furthermore there is no theoretical work on the influence of bidispersity on the balance between SmA and SmC.

In the existing theoretical approaches different models for the ordering in smectic A phases of molecules of substantially differing lengths are presented (Figure 11).

One possibility is to fill the space by nanosegregation of long and short mesogens. This segregation can be of the intra-layer (Figure 11a) or inter-layer (Figure 11b) type. Both options however are entropically unfavourable. In the intra-layer type all molecules are organized within one layer. Demixing of the two kinds of mesogens occurs locally inside the layers. Furthermore the layer spacing of such a smectic phase would - for all mole fractions - correspond to the length of the longer molecule (Figure 11a). However, in our SAXS-measurements we observed a layer spacing which varied linearly with the mole fraction and it was always smaller than the length of the long molecule. In the second kind of nanosegregation - the interlayer type - the two kinds of molecules demix and each of them forms their own layers. The 'layer spacing' observed by SAXS 
a)

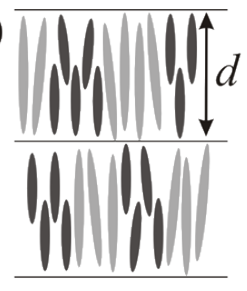

b)

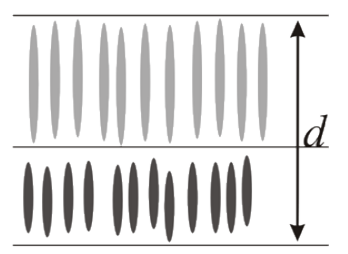

c)

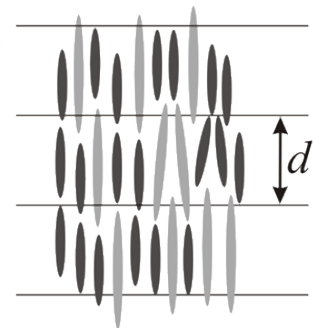

Figure 11: Schematic sketches of different models for smectic A ordering of bidisperse mesogens. Different models are: a) Intra-layer segregation, b) Inter-layer segregation or c) Out-of-layer fluctuations. After $[5,18]$.

(cf. repeating unit) for this kind of smectic phase would correspond to the added lengths of the two molecules (Figure 11b). As the experimentally found layer spacing is always smaller than the long molecule, this also cannot be the correct explanation.

The last possibility is a more dynamic picture, where the space is filled by out-of-layer fluctuations (Figure 11c). The long molecules organize themselves in the layers formed by the short molecules by out-of-layer fluctuations. The layer spacing of this kind of smectic A phases would be in between the lengths of the two molecules, while the quality of smectic ordering, e.g. the smectic order parameter $\Sigma$, would be essentially lowered in comparison to the pure compounds. The experimental findings for both the layer spacing and the smectic ordering are in complete agreement with this model (see Figure 4 and Figure 8).

With all this results we thus believe that out-of-layer fluctuations are the most realistic model to describe the structure of bidisperse smectics. This also explains the strong influence of bidispersity on the balance between SmA and SmC. Since SmA phases might tolerate out-of-layer fluctuations much more easily, a stabilization of SmA at the expense of the SmC phase might occur. Therefore, the structure of bidisperse smectics is signified by extensive out-of-layer fluctuations.

\section{Experimental}

Compounds 2-[4-(tetradecyloxy)phenyl]-5-(tetradecyloxy)pyrimidine (PhP14) [1], 6-[4-(butyloxy)phenyl]-3-(octyloxy)pyridazine $(6 \mathrm{PhPz})[3]$ and $(R, R)-2$-[4-(octyloxy)phenyl]-5- (2,3-difluorohexyloxy)pyridine (MDW510) [7] were synthesized according to published procedures and shown to have the expected physical and spectral properties. The liquid crystal 2-[4-(butoxy)phenyl]-5-(octyloxy)pyrimidine (2PhP) was obtained from a commercial source. X-Ray scattering experiments were performed with $\mathrm{Ni}$-filtered $\mathrm{CuK}_{\alpha}$ radiation (wavelength $1.5418 \AA$ ). Small angle scattering data from unaligned samples (filled into Mark capillary tubes of $0.7 \mathrm{~mm}$ diameter) were obtained using a Kratky compact camera (A. Paar) equipped with a temperature controller (A. Paar) and a one-dimensional electronic detector (M. Braun). For polarized optical microscopy a Leica DM-LP polarizing microscope with an Instec HS1-i hot stage was used. The optical tilt angles $\theta$ were determined by polarizing microscopy on samples aligned in rubbed nylon/ITO coated glass cells with a spacing of $1.6 \mu \mathrm{m}$ (AWAT PPW, Poland). To enable ferroelectric switching of the tilt direction $[19,20]$ in the achiral mixtures, $4 \mathrm{~mol} \%$ of the chiral dopant MDW510 was added to receive chiral SmC* phases. The measurements of $\theta$ were taken at a field strength $E$ of $12.5 \mathrm{~V} \mathrm{\mu m}^{-1}$ as half the rotation between the two optical extinction positions corresponding to opposite signs of $E$. A Netzsch DSC-204 Phoenix instrument was used for differential scanning calorimetry analyses at a scan rate of $5 \mathrm{~K} \mathrm{~min}^{-1}$.

\section{Acknowledgments}

We thank the Deutsche Forschungsgemeinschaft (DFG Gi 243/5) for financial support.

\section{References}

1. Hartley, C. S.; Kapernaum, N.; Roberts, J. C.; Giesselmann, F.; Lemieux, R. P. J. Mater. Chem. 2006, 16, 2329-2337. doi:10.1039/b515313a

2. Onsager, L. Ann. N. Y. Acad. Sci. 1949, 51, 627-659. doi:10.1111/j.1749-6632.1949.tb27296.x

3. Zaschke, H.; Hyna, C.; Schubert, H. Z. Chem. 1977, 17, 333-334.

4. Hegmann, T.; Meadows, M. R.; Wand, M. D.; Lemieux, R. P. J. Mater. Chem. 2004, 14, 185-190. doi:10.1039/b307673c

5. Diele, S. Ber. Bunsen-Ges. Phys. Chem. 1993, 97, 1326-1336.

6. The optical tilt angle is measured with a polarizing microscope in chiral $\mathrm{SmC}^{*}$ phases by ferroelectric switching of the tilt direction $[19,20]$.

7. Thurmes, W. N.; Wand, M. D.; Vohra, R. T.; Walba, D. M. Mol. Cryst. Liq. Cryst. 1991, 204, 1-7. doi:10.1080/00268949108046588

8. Thurmes, W. N.; Wand, M. D.; Vohra, R. T.; More, K. M.; Walba, D. M. Liq. Cryst. 1993, 14, 1061-1068. doi:10.1080/02678299308027814

9. These mixtures show a tilt angle which is smaller than $10^{\circ}$ over a temperature range of about $20 \mathrm{~K}$. This is quite unusual, as usual tilt angles of ferroelectric liquid crystals with second order $\mathrm{SmA}^{*}$ to $\mathrm{SmC}^{*}$ phase transitions are in the range of $\theta=20^{\circ}$ to $25^{\circ}$. Materials with first order phase transitions show tilt angles up to $\theta=45^{\circ}$.

10. Lagerwall, J. P. F.; Giesselmann, F. ChemPhysChem 2006, 7, 20-45. doi:10.1002/cphc.200500472 
11. The translational order parameter $\Sigma$ gives a measure for the quality of smectic layering. It is defined as the amplitude of the density wave arising from the periodic one-dimensional smectic layer structure [21, 22].

12. Kapernaum, N.; Giesselmann, F. Phys. Rev. E 2008, 78, No. 062701. doi:10.1103/PhysRevE.78.062701

13. Koden, M.; Anabuki, T.; Nakagawa, K.; Awane, K. Jpn. J. Appl. Phys., Part 2 1991, 30, L1129-L1131. doi:10.1143/JJAP.30.L1129

14. Stroobants, A. Phys. Rev. Lett. 1992, 69, 2388-2391. doi:10.1103/PhysRevLett.69.2388

15. Stroobants, A. J. Phys.: Condens. Matter 1994, 6, A285-A288. doi:10.1088/0953-8984/6/23A/045

16. Koda, T.; Kimura, H. J. Phys. Soc. Jpn. 1994, 63, 984-994. doi:10.1143/JPSJ.63.984

17. Bemrose, R. A.; Care, C. M.; Cleaver, D. J.; Neal, M. P. Mol. Phys. 1997, 90, 625-635. doi:10.1080/00268979709482645

18. Cinacchi, G.; Mederos, L.; Velasco, E. J. Chem. Phys. 2004, 121, 3854-3863. doi:10.1063/1.1774153

19. Bahr, C.; Heppke, G. Ber. Bunsen-Ges. Phys. Chem. 1987, 91 , 925-929.

20. Giesselmann, F.; Heimann, A.; Zugenmaier, P. Ferroelectrics 1997, 200, 237-256. doi:10.1080/00150199708008609

21. McMillan, W. L. Phys. Rev. A 1971, 4, 1238-1246. doi:10.1103/PhysRevA.4.1238

22. McMillan, W. L. Phys. Rev. A 1972, 6, 936-947. doi:10.1103/PhysRevA.6.936

\section{License and Terms}

This is an Open Access article under the terms of the Creative Commons Attribution License

(http://creativecommons.org/licenses/by/2.0), which permits unrestricted use, distribution, and reproduction in any medium, provided the original work is properly cited.

The license is subject to the Beilstein Journal of Organic Chemistry terms and conditions:

(http://www.beilstein-journals.org/bjoc)

The definitive version of this article is the electronic one which can be found at: doi:10.3762/bjoc. 5.65 